\title{
Longevidade de larvas infectantes de Haemonchus sp. em duas alturas de pasto de Brachiaria brizantha cv. Marandu
}

\author{
[Longevity of Haemonchus sp. infectant larvae in Brachiaria brizantha cv. Marandu pastures \\ kept under two heights]
}

\section{"Artigo Científico/Scientific Article"}

\author{
Paula Mara Ribeiro Troncha*, Manoel Eduardo Rozalino Santos, Rosilene de Fátima Moreira \\ Mota, Ariel Mendes de Resende, Fernanda Rosalinski-Moraes
}

Faculdade de Medicina Veterinária e Zootecnia, Universidade Federal de Uberlândia (UFU), Uberlândia-MG, Brasil. *Autor para correspondência/Corresponding author: E-mail: paulartvet@gmail.com

\begin{abstract}
Resumo
O objetivo deste trabalho foi quantificar a recuperação de larvas infectantes de Haemonchus sp. em capim Marandu (Brachiaria brizantha), no período da seca (inverno) e das chuvas (verão) ao longo do tempo; bem como verificar se a altura do pasto influenciou neste tempo de sobrevivência. O experimento foi conduzido em Uberlândia, durante os meses de agosto a novembro de 2014 (seca) e janeiro a março de 2015 (chuvas). Foram considerados dois tratamentos (alturas de pasto 10 e $35 \mathrm{~cm}$ ), com coleta de amostras nos dias $0,7,14$, $28,42,56$ e 70 dias. Houve contaminação experimental de parcelas com fezes de caprinos com média de 1600 ovos de estrongilídeos por grama (OPG). Foi realizada amostragem por cortes dos estratos inferior e superior do capim. Houve maior número de larvas recuperadas no estrato inferior no inverno $(p<0,05)$. Não houve diferença no número de larvas recuperadas dos diferentes estratos no verão, nem entre as diferentes alturas de pasto $(\mathrm{p}>0,05)$. Foram encontradas larvas até o $42^{\circ}$ dia no período chuvoso e até o fim do trabalho durante a seca, com maior quantidade de larvas $(\mathrm{p}<0,05)$ no $7^{\circ}$ e $14^{\circ}$ dia pós-contaminação. Pode se inferir a necessidade de estudos para maior conhecimento da influência das variáveis microclimáticas do pasto nas larvas infectantes de nematódeos.
\end{abstract}

Palavras-chave: larva de terceiro estádio; microclima; migração; parasita gastrintestinal.

\begin{abstract}
The objective of this work was to quantify the number of Haemonchus sp. infective larvae recovered from Marandu grass (Brachiaria brizantha) during the drought (winter) and the rainy season (summer) over time; as well as to check if the height of the pasture influences in this survival period. The experiment was conducted in Uberlândia, Minas Gerais State, Brazil, from August to November 2014 (drought) and January to March 2015 (rain). Two treatments were considered the pasture heights of 10 and of $35 \mathrm{~cm}$, with sampling collection on days $0,7,14,28,42,56$, and 70 after contamination of the parcels with goat feces with an average of 1600 of Strongyle eggs per gram (EPG). Sampling was performed by cutting the lower and upper strata of the grass. There were greater number of larvae recovered in the lower stratum in winter $(p<0.05)$. There was no significant difference in the number of larvae recovered from the different strata during the summer and variation in the amount of larvae over the days. There was no difference in the number of larvae in the different strata during the rainy season, nor in pastures kept at different heights ( $>>0.05)$. Larvae were found until the $42^{\text {nd }}$ day in the rainy period and until the end of the study during the drought, with greater quantity of larvae $(\mathrm{P}<0.05)$ on the 7 th and 14th day after contamination. The need for further knowledge of how microclimatic variables can influence larval survival on the pasture may be inferred.
\end{abstract}

Keywords: microclimate; migration; gastrointestinal parasites; third-stage larvae. 


\section{Introdução}

Um dos maiores problemas que afeta a produção de ovinos e caprinos no Brasil e no mundo está relacionado às questões sanitárias, principalmente as helmintoses gastrintestinais. Os principais parasitos gastrintestinais de pequenos ruminantes são da ordem Strongylida e, na região Sudeste do Brasil, os mais importantes são Haemonchus contortus e Trichostrongylus colubriformis, seguidos por Cooperia curticei e Oesophagostomum columbianum (Wilmsen et al., 2014).

O ciclo evolutivo desses parasitos compreende duas fases. Na fase de vida livre, os ovos são eliminados para o meio junto com as fezes do hospedeiro. As larvas eclodem, passam por duas mudas e se tornam L3 (estádio infectante). O hospedeiro se infecta ao ingerir este estádio juntamente com o pasto, dando início à fase de vida parasitária (Sotomaior et al., 2009). Durante a fase de vida livre, as condições do meio, tais como a umidade, radiação ultravioleta, temperatura e oxigenação são determinantes do tempo de sobrevivência e longevidade dos estádios de vida não parasitários (O’Connor et al., 2006). Desta forma, a possibilidade de alterar o microclima entre solo e pasto pelo manejo de pastejo e espécie forrageira utilizada pode ser uma ferramenta de controle parasitário.

Dentre as gramíneas forrageiras utilizadas no Brasil, Brachiaria brizantha é a espécie mais amplamente distribuída, sobretudo na região dos Cerrados. Segundo Valle et al. (2010), o cultivar Marandu corresponde a cerca de $50 \%$ das pastagens cultivadas no Brasil. Visando conhecer melhor os fatores que possam interferir na longevidade e viabilidade das larvas infectantes de estrongilídeos gastrintestinais em condições de pastagens no Cerrado brasileiro, este trabalho foi desenvolvido para: (1) quantificar a recuperação de L3 em capim Marandu (Brachiaria brizantha) no período da seca e de chuvas na região de Uberlândia - MG; (2) estimar o tempo pelo qual as larvas permaneceram viáveis na pastagem e (3) verificar se a altura do pasto influenciou este tempo de sobrevivência.

\section{Material e Métodos}

$\mathrm{O}$ experimento foi realizado na Fazenda Experimental do Capim Branco, Universidade Federal de Uberlândia, no Estado de Minas Gerais. A área encontra-se $776 \mathrm{~m}$ acima do nível do mar e as coordenadas geográficas aproximadas do local são $18^{\circ} 30^{\prime}$ de latitude sul e $47^{\circ} 50^{\prime}$ de longitude oeste. O período experimental da seca foi de agosto a novembro de 2014 e o chuvoso, de janeiro a março de 2015.

Foram separados dois piquetes de $800 \mathrm{~m}^{2}$ cada um, com pastagem de Brachiaria brizantha cv. Marandu previamente estabelecida. A fim de manter a morfologia do pasto mais próxima possível das condições naturais de ocupação, esta área foi pastejada regularmente por ovinos até 30 dias antes do início do experimento. $\mathrm{O}$ delineamento experimental foi composto por dois tratamentos, sendo alturas de pasto de 10 e $35 \mathrm{~cm}$, com coleta de amostras nos dias $0,7,14,28,42,56$ e 70 dias. Como as análises foram realizadas em triplicata para cada data de coleta, foram totalizados 42 sítios de coleta por período, que foram demarcados pelo uso de palitos de madeira. Posteriormente, foi procedida à contaminação experimental de cada parcela pelo depósito de cinco gramas de fezes com média de 1600 ovos de estrongilídeos por grama de fezes (OPG). Para quantificar o número de $\mathrm{OPG}$ foi realizado o método de Gordon e Whitlock (1939), com sensibilidade para 50 OPG.

Estas fezes foram obtidas a partir de caprinos portadores de infecção natural. Foram pesadas cinco gramas de um pool de fezes de caprinos doadores em copos descartáveis. As fezes foram umedecidas e as cíbalas desagregadas, acrescidas de vermiculita e incubadas à temperatura ambiente por sete dias, a fim de que os ovos evoluíssem até larvas de terceiro estádio (L3). O conteúdo remanescente de cada copo foi utilizado para contaminar o centro de cada unidade experimental no D0.

As amostras de pasto foram obtidas sempre às 8:00 horas, com auxílio de um amostrador quadrado com $10 \mathrm{~cm}$ de lado, posicionado de forma a conter o local da deposição do bolo fecal em seu centro. Todo o pasto presente no amostrador foi cortado rente ao solo, sendo realizado no próprio local a divisão de estrato superior e inferior a partir do ponto médio da altura do capim. Também foi colhida a matéria morta (serrapilheira) contida na área de coleta, que foi analisada juntamente com as fezes presentes nesta porção. A altura do capim no sítio de coleta foi mensurada com uma régua, posicionada no centro do amostrador e perpendicular ao solo. Para monitoramento diário das condições climáticas, foi posicionado um termômetro para temperaturas máximas e mínimas e um pluviômetro junto à área experimental. 
Para obtenção das larvas a partir das amostras de pasto e fezes em laboratório, foi adaptado o método de Rugai et al. (1954), que se baseia no termo e hidrotropismo das larvas de nematoides. As larvas infectantes de estrongilídeos foram quantificadas em microscópio óptico e identificadas conforme a chave morfométrica proposta por Van Wyk et al. (2004).

As análises estatísticas foram realizadas de forma separada para cada período do ano. Para verificar se houve diferença entre o número de larvas recuperadas nos diferentes estratos e nos dois tratamentos ( $10 \mathrm{~cm}$ e $35 \mathrm{~cm}$ de altura) os dados foram analisados pelos testes de Wilcoxon $(\mathrm{p}<0,05)$ e Mann Whitney $(\mathrm{p}<0,05)$, respectivamente. Para verificar se houve diferença no número de L3 entre os dias de coleta $(0,7,14$, $28,42,56$ e 70 dias) foi aplicado o teste de KruskalWallis seguido do teste de Dunn, utilizando o software Instat 3.06.

\section{Resultados}

Durante o período experimental foi observado o crescimento gradual do capim. No período da seca, este aumento foi menos expressivo, sendo que as parcelas com 10 e $35 \mathrm{~cm}$ apresentaram média de 16,22 e $34,66 \mathrm{~cm}$ no $70^{\circ}$ dia de experimento. Para o período chuvoso, esses valores foram de 41,8 e 121,6 cm, respectivamente.

Foi possível recuperar das amostras de pasto e fezes remanescentes sobre os sítios de coleta larvas infectantes de Haemonchus sp. e Trichostrongylus sp. Porém, apenas as larvas de Haemonchus sp. foram obtidas em quantidades significativas para proporcionar análise estatística e terão seus resultados apresentados. Nas coletas controle (dia zero), não foi encontrada nenhuma larva de estrongilídeo nas amostras de pasto, nem na estação seca e nem na chuvosa.

A recuperação de larvas de Haemonchus sp. foi maior no estrato inferior em relação ao superior no período seco $(\mathrm{p}<0,05)$, mas não no período das chuvas (Tabela 1). Embora um maior número de L3 tenha sido recuperado no pasto mantido a $10 \mathrm{~cm}$, não foi considerada significativa esta diferença (Tabela 2). O mesmo se deu para o número de larvas recuperadas a partir das amostras de fezes (Tabela 3).

Ao observar o número de larvas infectantes encontradas por dia de coleta e estrato do pasto (Figura 1), verificou-se que a maior quantidade de L3 foi encontrada no sétimo dia pós contaminação (D7). Este número decresceu gradualmente em todos os estratos pesquisados, manifestando diferença significativa a partir do D28. No período das águas, não foi mais possível recuperar larvas de Haemonchus sp. em nenhum estrato coletado e nenhuma parcela a partir do D56.

Em relação às variações de temperatura (máxima e mínima) no período seco do ano, foi possível observar grande amplitude térmica diária. As temperaturas máximas foram acima dos $30^{\circ} \mathrm{C} \mathrm{e}$ as mínimas variaram de 10 a $15^{\circ} \mathrm{C}$ até o mês de setembro, subindo para $20^{\circ} \mathrm{C}$ no mês de outubro de 2014 (Figura 2). A pluviosidade esteve próxima de $0 \mathrm{~mm}$ de chuva na maior parte deste período experimental, sendo o esperado para esta época do ano. O período chuvoso esteve de acordo com o previsto com temperaturas mais altas, menor amplitude térmica diária ao se comparar com o período seco e chuvas bem distribuídas.

Tabela 1. Média, desvio padrão, valor máximo e valor mínimo do número de larvas infectantes (L3) de Haemonchus sp. obtidas no estrato superior e inferior em área cultivada com Brachiaria brizantha cv. Marandu, entre agosto e novembro de 2014 (seco) e janeiro a março de 2015 (chuvoso) em Uberlândia-MG.

\begin{tabular}{ccc}
\hline & $\mathbf{N}^{\mathbf{0}}$ L3 Haemonchus sp. Estrato Superior & $\mathbf{N}^{\mathbf{O}} \mathbf{L 3}$ Haemonchus sp. Estrato Inferior \\
\hline \multicolumn{2}{c}{ Período seco } \\
\hline Média & $1,095 \mathrm{~B}$ & $1,738 \mathrm{~A}$ \\
Desvio padrão & 2,621 & 3,284 \\
Valor máximo & 10 & 12 \\
Valor mínimo & 0 & 0 \\
\hline & Período chuvoso \\
\hline Média & $1,853 \mathrm{~A}$ & $1,529 \mathrm{~A}$ \\
Desvio padrão & 3,375 & 3,305 \\
Valor máximo & 19 & 14 \\
Valor mínimo & 0 & 0 \\
\hline
\end{tabular}

*Letras diferentes na mesma linha indicam diferença significativa pelo teste de Wilcoxon $(\mathrm{p}<0,05)$. 
Tabela 2. Média, desvio padrão, valor máximo e valor mínimo do número de larvas infectantes (L3) de Haemonchus sp. recuperadas no estrato superior e inferior de capim nos pontos marcados em área cultivada com Brachiaria brizantha cv. Marandu nas alturas $10 \mathrm{~cm}$ e $35 \mathrm{~cm}$, contaminadas com fezes de caprinos, entre agosto e novembro de 2014 (seco) e janeiro a março de 2015 (chuvoso) em Uberlândia-MG.

\begin{tabular}{|c|c|c|c|c|}
\hline & n. ${ }^{\circ}$ L3 no & Superior & n. ${ }^{\circ} \mathrm{L} 3 \mathrm{nc}$ & Inferior \\
\hline & $10 \mathrm{~cm}$ & $35 \mathrm{~cm}$ & $10 \mathrm{~cm}$ & $35 \mathrm{~cm}$ \\
\hline \multicolumn{5}{|c|}{ Período seco } \\
\hline Média & $0,761 \mathrm{~A}$ & $1,429 \mathrm{~A}$ & $1,819 \mathrm{~A}$ & $1,667 \mathrm{~A}$ \\
\hline Desvio padrão & 2,427 & 2,821 & 2,816 & 2,745 \\
\hline Valor máximo & 9 & 10 & 12 & 9 \\
\hline Valor mínimo & 0 & 0 & 0 & 0 \\
\hline \multicolumn{5}{|c|}{ Período chuvoso } \\
\hline Média & $2,375 \mathrm{~A}$ & $1,389 \mathrm{~A}$ & $1,563 \mathrm{~A}$ & $1,500 \mathrm{~A}$ \\
\hline Desvio padrão & 4,829 & 2,453 & 3,687 & 3,034 \\
\hline Valor máximo & 19 & 8 & 14 & 12 \\
\hline Valor mínimo & 0 & 0 & 0 & 0 \\
\hline
\end{tabular}

*Letras indicam resultado do teste de Mann Whitney para dados agrupados na mesma linha e estrato pesquisado $(\mathrm{p}<0,05)$.

Tabela 3. Média, desvio padrão, valor máximo e valor mínimo do número de larvas infectantes (L3) de Haemonchus sp. recuperadas na matéria morta (fezes), nos pontos marcados em área cultivada com Brachiaria brizantha cv. Marandu nas alturas $10 \mathrm{~cm}$ e $35 \mathrm{~cm}$ contaminadas por coprocultura no dia “zero", entre agosto e novembro de 2014 (seco) e janeiro a março de 2015 (chuvoso) em UberlândiaMG.

\begin{tabular}{lcc}
\hline & Altura da cultivar & \\
\hline & $\mathbf{1 0} \mathbf{~ c m}$ & $\mathbf{3 5} \mathbf{~ c m}$ \\
\hline Média & Período seco & \\
Desvio padrão & $17,714 \mathrm{~A}$ & $22,381 \mathrm{~A}$ \\
Valor máximo & 31,967 & 41,924 \\
Valor mínimo & 103 & 165 \\
\hline & 0 & 0 \\
\hline Média & Período chuvoso & $0,1667 \mathrm{~A}$ \\
Desvio padrão & $0,1250 \mathrm{~A}$ & 0,5145 \\
Valor máximo & 0,3416 & 2 \\
Valor mínimo & 1 & 0 \\
\hline *Letras indicam resultado do teste de Mann Whitney para comparação de dado na mesma linha $(\mathrm{p}<0,05)$.
\end{tabular}
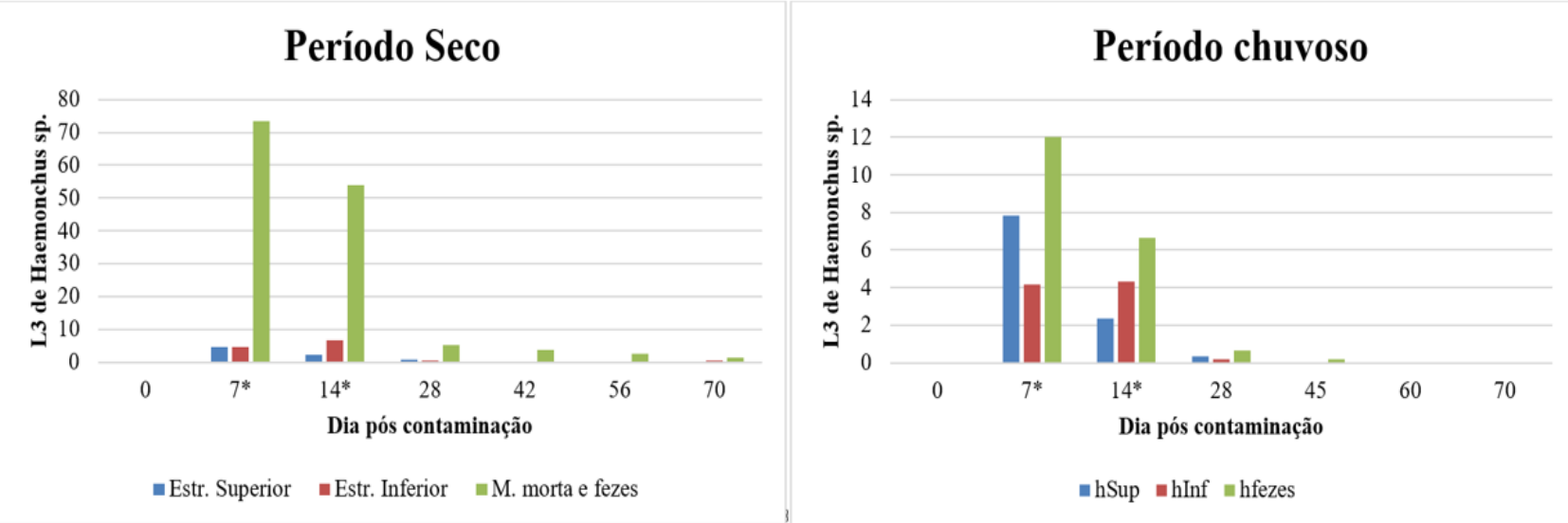

Figura 1. Média do número de larvas infectantes (L3) de Haemonchus sp. recuperadas nos diferentes dias de coleta dos pontos marcados em área cultivada com Brachiaria brizantha cv. Marandu, conforme estrato coletado e época do ano em Uberlândia-MG. $*(\mathrm{P}<0,05)$ pelo teste de Kruskal-Wallis, seguido do teste de Dunn. 

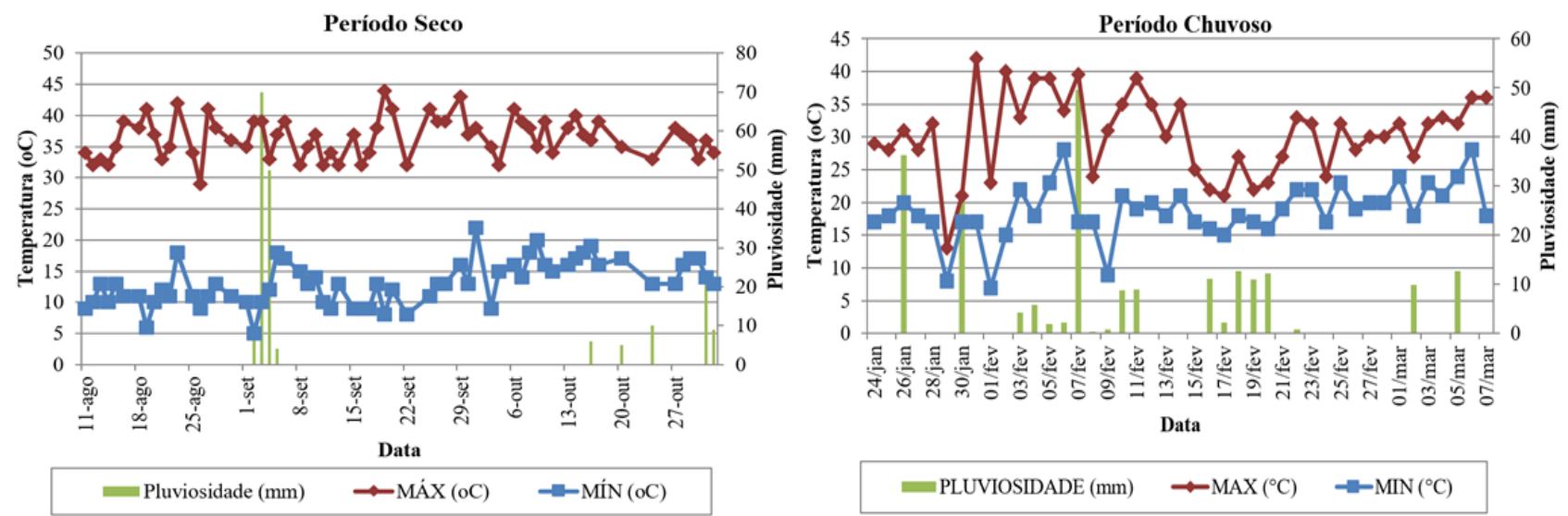

Figura 2. Temperatura máximas (ToC máx), mínimas (ToC min) e pluviosidade $(\mathrm{mL})$ ao longo do período de recuperação de larvas infectantes de Haemonchus sp. (L3) em pastos de Brachiaria brizantha cv. Marandu, na Fazenda Capim Branco, em Uberlândia.

\section{Discussão}

Nas condições experimentais em que foi desenvolvido o presente trabalho, foi possível recuperar larvas infectantes de Haemonchus sp. até o último dia de coleta após a contaminação das parcelas no período da seca e até o $45^{\circ}$ dia póscontaminação no período chuvoso. Este tempo se assemelha ao obtido por outros autores. Dittrich et al. (2004) recuperaram L3 no pasto de Paspalum notatum até 30 dias após contaminação das parcelas, durante o verão no estado do Paraná; Almeida et al. (2005) o fizeram até a $15^{\mathrm{a}}$ semana do início do experimento, trabalhando no Estado do Rio de Janeiro durante a seca.

Apesar do longo período pelo qual foi possível recuperar as larvas infectantes a partir das amostras de pasto, foi possível observar uma redução significativa dessa recuperação após o $14^{\circ}$ dia. Rocha et al. (2008) também obtiveram valor máximo de recuperação de L3, tanto das fezes como do pasto, duas semanas após a contaminação experimental, durante o verão. No trabalho de Rocha et al. (2012), o pico na recuperação de L3 ocorreu na $4^{\text {a }}$ semana de avaliação, em momento experimental realizado do outono ao inverno em parcelas de $30 \mathrm{~cm}$ de altura, tanto na Brachiaria decumbens, quanto na de Panicum maximum, no Estado de São Paulo. Este dado pode ser interessante, uma vez que pastagens manejadas sob lotação intermitente necessitam de um período superior a 14 dias para recuperação da área foliar após a ocupação.

No presente trabalho, foi possível recuperar mais larvas infectantes no estrato inferior do capim durante a seca (Tabela 1). Possivelmente as condições de microclima deste estrato associadas à temperatura e umidade fecal facilitaram a permanência das larvas (O'Connor et al., 2006). No período das águas (janeiro a março) não houve diferença na recuperação de L3 nos diferentes estratos, sendo observado aumento discreto na média de larvas recuperadas no estrato superior na altura de $10 \mathrm{~cm}$. Santos et al. (2012), trabalhando na região de Botucatu, São Paulo, encontraram maior número de larvas recuperadas no estrato superior (acima de $20 \mathrm{~cm}$ ) de Brachiaria decumbens durante o verão, atingindo pico no sétimo dia após contaminação das parcelas. Os autores atribuíram à chuva a capacidade de dissolução das fezes, proporcionando um filme úmido para a migração das larvas até as porções superiores do capim.

Ao comparar o capim mantido em diferentes alturas (10 e $35 \mathrm{~cm}$ de altura), não foi observada diferença em relação à quantidade de larvas de Haemonchus sp. encontradas nas fezes e na pastagem, tanto no período da seca quanto das águas. Dittrich et al. (2004) realizaram dois experimentos na região metropolitana de Curitiba, Paraná, utilizando as forragens Paspalum paniculatum, Cynodon sp. (Tifton 85), Azevém e Aveia, nos períodos de verão e inverno e afirmaram que a altura das plantas não foi determinante na quantidade de larvas recolhidas.

Outros autores (Carneiro e Amarante, 2008; Rocha et al., 2008; Rocha et al., 2012), ao trabalharem com Brachiaria decumbens cv. Australiana, Cynodon dactylon cv. Coast-cross e Panicum maximum cv. Aruana, mantidas nas alturas de 5 e $30 \mathrm{~cm}$, na região de Botucatu, São Paulo, puderam recuperar mais larvas de terceiro estádio nas parcelas de $30 \mathrm{~cm}$ em determinados momentos de seus experimentos. Isso foi atribuído ao fato de que a maior densidade de massa 
forrageira, que ocorre quando o pasto é mantido em maiores alturas, possibilita a formação de microclimas favoráveis à sobrevivência de larvas. É possível, portanto, que se houvesse um rebaixamento do capim Marandu até a altura de 5 $\mathrm{cm}$ talvez pudesse ser verificada esta diferença. No entanto, este estudo seria de pouca aplicação prática, uma vez que esta altura representaria a remoção quase completa da massa foliar do capim e das gemas apicais. Silva (2013) relata que o melhor consumo diário do capim Marandu em condições de pastejo seja entre 30 e $40 \mathrm{~cm}$.

A variabilidade na recuperação de larvas de nematódeos no ambiente está associada a diversos fatores que influenciam na migração e sobrevivência nos estádios larvais. O'Connor et al. (2006) relataram que o desenvolvimento e viabilidade larval estão relacionados a intervalos ideais de umidade e temperatura disponível na fase de vida livre. Alterações no macroclima (temperatura ambiental, umidade do ar, luminosidade e pluviosidade) e no microclima (umidade e temperatura fecal) podem influenciar fortemente a sobrevida das larvas no pasto em seus diferentes estádios.

Em condições tropicais, a sobrevida das larvas infectantes tende a ser menor do que em clima temperado, pois temperaturas altas aumentam o metabolismo das larvas, causando um esgotamento rápido de suas reservas energéticas (Cheah e Rajamanickam, 1997). A exposição à luz ultravioleta no período do verão também pode ser prejudicial para a sobrevivência larval (Santos et al., 2012).

Em períodos de escassez de chuva, como o observado no presente trabalho durante o período da seca, os nematoides podem entrar em processo de anidrobiose, ou seja, suspender temporariamente suas funções vitais e voltar à atividade metabólica quando reidratados. Esse fenômeno explica um pico de contaminação das pastagens após períodos de seca e pluviosidade, garantindo a sobrevivência das larvas por um longo período (Rocha et al., 2012).

Alguns trabalhos mostraram uma recuperação de larvas maior no período seco do ano (Dittrich et al., 2004; Rocha et al., 2007; Rocha et al., 2012) sugerindo uma capacidade de sobrevivência das larvas a períodos secos e de reidratação sem efeitos deletérios (Rocha et al., 2012).

Em estudo realizado no período das chuvas, houve baixa recuperação de larvas tanto no bolo fecal quanto na forragem, independente da altura. Esse fato pode ser explicado pela intensa precipitação que ocorreu logo após a contaminação da pastagem, levando a uma lixiviação das larvas e diluição no pasto, extravazando a área da parcela recolhida (Rocha et al., 2012).

Rocha et al. (2007) não conseguiram recuperar nenhuma larva tanto no inverno quanto no verão, em trabalho realizado com capins Brachiaria decumbens (cv. Australiana) e Panicum maximum (cv. Aruana), em parcelas com altura de $30 \mathrm{~cm}$, avaliados ao $10^{\circ}$ dia após contaminação das parcelas. Os autores atribuíram à falta de chuva no dia da contaminação, interferindo na migração e até mesmo na sobrevivência das larvas nas fezes.

A migração de larvas e a sobrevivência no ambiente precisam de estudos mais detalhados diante da variabilidade de fatores que estão associados e, inclusive, à escala de importância de um efeito sobre outro.

\section{Conclusão}

O presente trabalho mostra uma recuperação de larvas em até 70 dias após a contaminação das amostras de pasto no período seco e até 45 dias após contaminação no período chuvoso. Não há diferença no número de larvas obtidas entre os dosséis de Brachiaria brizantha cv. Marandu com alturas iniciais de 10 e $35 \mathrm{~cm}$, mas há maior acúmulo de larvas infectantes no estrato inferior do capim durante o período da seca.

\section{Conflito de Interesse}

Os autores declararam não existir conflitos de interesse.

\section{Referências}

Almeida, L.R.; Castro, A.A.; Silva, F.J.M.; Fonseca, A.H. Desenvolvimento, sobrevivência e distribuição das larvas infectantes de nematoides gastrintestinais de ruminantes, na estação seca da baixada fluminense, RJ. Revista Brasileira de Parasitologia Veterinária, 14(3): 89-94, 2005.

Carneiro, R.D.; Amarante, A.F.T. Seasonal effect of three pasture plants species on the freeliving stages of Haemonchus contortus. Arquivo Brasileiro de Medicina Veterinária e Zootecnia, 60(4): 864-872, 2008.

Cheah, T.S.; Rajamanickam, C. Epidemiology of gastro-intestinal nematodes of sheep in wet 
tropical conditions in Malaysia. Tropical Animal Health Production, 29:165-173, 1997.

Da Silva, S.C.; Gimenes, F.M.A.; Sarmento, D.O.L; Sbrissia, F.A.; Oliveira, D.E.; Hernandez-Garay, A.; Pire, A.V. Grazing behaviour, herbage intake and animal performance of beef cattle heifers on marandu palisade grass subjected to intensities of continuous stocking management. Journal of Agricultural Science, 151(5): 727-739, 2013.

Dittrich, J.R.; Gazda, T.L.; Piazzeta, R.G.; Rodrigues, C.S. Localização de larvas L3 de helmintos gastrintestinais de ovinos nas plantas forrageiras: efeito da altura e da espécie vegetal. Archives of Veterinary Science, 9(2): 43-48, 2004.

Gordon, H.M.; Whitlock, H.V. A new technique for counting nematode eggs in sheep faeces. Journal of the Council for Scientific and Industrial Research, 12: 50-52, 1939.

O'Connor, J.L.; Walkedn-Brown, W.S.; Kahn, L.P. Ecology of the free-living stages of major trichostrongylid parasites of sheep. Veterinary Parasitology, 142: 1-15, 2006.

Rocha, R.A.; Bricarello, P.A.; Rocha, G.P.; Amarante, A.F.T. Recuperação de larvas de Trichostrongylus colubriformis em diferentes estratos de Brachiaria decumbens e Panicum maximum. Revista Brasileira de Parasitologia Veterinária, 16(2):77-82, 2007.

Rocha, R.A.; Rocha, G.P.; Bricarello, P.A.; Amarante, A.F.T. Recuperação de larvas infectantes de Trichostrongylus colubriformis em três espécies de gramíneas contaminadas no verão. Revista Brasileira de Parasitologia Veterinária, 17(4): 227-234, 2008.

Rocha, R.A.; Rocha, G.P.; Bricarello, P.A.; Amarante, A.F.T. Recovery of
Trichostrongylus colubriformis infective larvae from three grass species contaminated in the autumn. Revista Brasileira de Parasitologia Veterinária, 21(4): 372-378, 2012.

Rugai, E.; Mattos, T.; Brisola, A.P. Nova técnica para isolar larvas de nematóides das fezesmodificação do método de Baermann. Revista do Instituto Adolfo Lutz, 14: 5-8, 1954.

Santos, M.C.; Silva, B.F.; Amarante, A.F.T. Environmental factors influencing the transmission of Haemonchus contortus. Veterinary Parasitology, 188: 277-284, 2012.

Sotomaior, C.S.; Rosalinski-Moraes, F.; Souza, F.P.; Milczewski, V.; Pasqualin, C.A. Parasitoses gastrintestinais dos ovinos e caprinos: alternativas de controle. Curitiba EMATER, 2009. Disponível em: http://www.arcoovinos.com.br/images/artigos Tecnicos/Parasitoses\%20Gastrintestinais\%20 dos\%20Ovinos \%20e\%20Caprinos.pdf. Acesso em: 25 fev. 2018.

Valle, C.B. do; Macedo, M.C.M.; Euclides, V.P.B.; Jank, L.; Resende, R.M.S. Gênero Brachiaria. In.: Fonseca DM; Martuschello JA. Plantas forrageiras. Viçosa: 2010. p.30-77.

Van Wyk, J.A.; Cabaret, J.; Michael, L.M. Morphological identification of nematode larvae of small ruminants and cattle simplified. Veterinary Parasitology, 119: 277-306, 2004.

Wilmsen, M.O.; Fernanda, B.; Bassetto, C.C.; Amarante A.F.T. Infecções por nematódeos gastrintestinais em ovinos criados em Botucatu, estado de São Paulo, Brasil. Revista Brasileira de Parasitologia Veterinária, 23(3):348-354, 2014. 